\title{
Conversations with a neurosurgeon
}

\section{Interview with Dr Fawaz Siddiqi}

\author{
Han Yan (Meds 2017), Ramona Neferu (Meds 2018) \\ Faculty Reviewer: Dr Fawaz Siddiqi, MD, MBA, FRCSC, FACS (Department of Clinical Neurological Sciences)
}

In the third of three interviews in this issue, we speak to Dr Fawaz Siddiqi. Dr Siddiqi is an Assistant Professor of Neurosurgery and Orthopaedics at Western University. He is the president of the Professional Service Organization and sits on the Medical Advisory Committee as well as the London Health Sciences Centre Board of Directors. He is also the course chair for the Healthcare Systems course at the Schulich School of Medicine and Dentistry.

UWOMJ: Tell us a bit about yourself, your career path and your education path.

Fawaz Siddiqi: I did my undergraduate degree in biochemistry at the University of Ottawa and came to Western for medical school. I did my neurosurgery residency training and an orthopaedic spine fellowship here, after which I came on as faculty. Clinically, I'm one of four spine surgeons in our combined neurosurgery and orthopaedic spine program at Victoria Hospital in London. My practice encompasses degenerative spinal conditions, oncology, and trauma.

What made you interested in neurosurgery? What makes it unique?

My attraction to neurosurgery happened relatively early in my career. I spent my summers after first and second year of medical school with the neurosurgical service at a hospital back home in Ottawa. Spending time with the neurosurgical team helped me determine whether it was something I wanted to do.

Neurosurgery is a challenging field to work in and it attracts people who really crave that sort of challenge. The procedures and patient population are very diverse. Neurosurgeons perform many procedures to help people with a variety of conditions. Neurosurgeons help with chronic pain through spinal cord stimulation, with movement disorders such as Parkinsonism through the use of deep brain stimulation, and curing epilepsy with surgical resections. In addition, we deal with oncological conditions of the brain and spine as well as vascular conditions, such as cerebral aneurysms and vascular malformations.

Spinal surgery itself is very rewarding. Patients come in who have a lot of difficulty walking and they can walk again after the operation. That's not something that a lot of specialties can lay claim to.

How would you compare neurology, psychiatry, and neurosurgery?

While the pathologies may overlap, the objectives and practice patterns of the practitioners are very different. That said, there is a huge amount of crossover between neurosurgery and neurology in terms of pain management, treatment for movement disorders, and epilepsy. Surgeons who work in those fields usually work in interdisciplinary teams. Epilepsy is a perfect example. You can't be an effective epilepsy surgeon if you don't have an effective epilepsy neurology partner. The same is true with movement disorder work. The bulk of my collaboration with other physicians is with oncologists and neurologists. As for psychiatry, there are more and more data suggesting that stimulation of certain areas of the brain, namely the areas of the limbic system and the cingulate gyrus, can actually help with depression as well.

Can you tell us a little bit about your Master of Business Administration (MBA) or other academic or personal interests you have?

During training, neurosurgical residents usually get a research/ elective period of about 9 months. Most people will use that to do a master's degree of some sort or a research project or an elective. When I looked at the breadth of options available to me, the MBA seemed to be the most effective degree in looking at healthcare systems operationally and being able to work through some of the problems that we have such as delivery of care to our patients.

For a physician who is involved in management, it can be a very powerful tool to have in your back pocket. I encourage people to look into it as a professional career option as the healthcare system evolves and encounters new difficulties, whether it be a funding perspective or from a structural perspective. We are going to need more and more physician voices involved in these issues.

One of the current projects that I am involved with at an institutional level is collaborating on working towards achieving better physician participation in healthcare management. Since the bulk of healthcare costs actually are driven by the physician's pen, physicians need to be more engaged in healthcare institution management in order to achieve efficient healthcare delivery. Traditionally, physicians worked as islands in most hospitals and clinics with very little oversight or participation in attempting to make the system better. This archaic model has to change.

What do you think the challenges are in neurosurgery in the next twenty years?

The outcomes from primary brain tumour treatment haven't changed much in the last number of years, and the prognosis remains pretty dismal for some types of brain tumours (12-14 months median survival with maximal therapy for primary high-grade glioma). So, from a research and innovation perspective, the causative factors of primary brain tumours still remains elusive. 
The other challenge is really delivery of care. The wait list for a spinal surgery can often times reach two years for consultation, and an additional year for surgery. The way we deliver consultative services to patients has to change. We can't rely on the traditional model where you have a physiotherapist, chiropractor, or family physician refer to a surgeon in isolation. We really need an interdisciplinary model of care for this specific patient population that will shorten wait times and improve evidence-based care.

\section{What's new and exciting in your field of neurosurgery?}

We are achieving a much better understanding of spinal biomechanics and becoming better at treating spinal diseases, whether minimally invasively or with open surgery. There is increasing awareness of the role of neurosurgery for epilepsy surgery, as well as for Parkinsonism, movement disorders, and chronic pain conditions.

Do you have any comments about future job prospects in neurosurgery in Canada and elsewhere?

We have not had enough jobs in Canada to sustain the number graduating from neurosurgery, so many neurosurgeons found jobs in the United States after training. Most centres in the US are still hiring Canadian-trained neurosurgeons and recognizing the Canadian training. In my year, we had three of us stay in Canada and everyone else went to the US, and as far as I know, everyone is employed and has fantastic jobs.

\section{Are there opportunities for working abroad for medical relief purposes? \\ Organizations tend to recruit pediatric neurosurgeons due to} the high burden spina bifida and hydrocephalus in these nations. There is a role for surgeons in medical education abroad through entities such as the American College of Surgeons.
How is the balance between family time, call-time, and day-today work?

All twelve members in our faculty are married, and most of us have children. I think achieving a work-life balance is very manageable. The Victoria Hospital neurosurgeons have a call frequency of 1 in 4 and we do call a week at a time, but I usually go home around 6:30 PM, and am back for meetings around 7:00 AM. In general, I'm not up every single night operating, and we have a good vacation roster. Gone are the days where people are living in the hospitals and not seeing their families.

If a medical student is interested in neurosurgery, what advice would you have for them?

The best thing to do is really explore the specialty through clinical electives and spend time with the neurosurgical service. That will give you the best perspective as to what the practice looks like and the pathology that you will be dealing with on a regular basis. It's harder if you haven't had that experience to look at it as a viable specialty option because you really haven't experienced what it's like and you have only an abstract concept of what a neurosurgeon might do. But that's really advice that I give for anybody who is looking at any specialty. 